\title{
Advances in Retinal Disease Treatment and Diagnosis 2017
}

\author{
An Expert Interview with Maurizio Battaglia Parodi \\ Department of Ophthalmology, Ospedale San Raffaele, University Vita-Salute, Milan, Italy
}

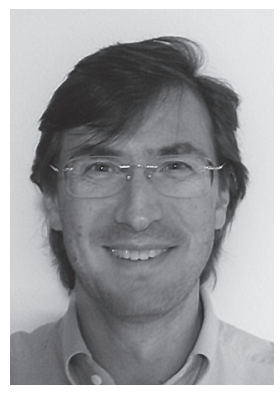

DOI: https://doi.org/10.17925/EOR.2017.11.02.90

Maurizio Battaglia Parodi

Maurizio Battaglia Parodi is currently working at the Department of Ophthalmology, Ospedale San Raffaele in Milan, and is Associate Professor at University Vita-Salute in Milan. He has had previous appointments in the Departments of Ophthalmology at the University of Trieste and the University of Udine, Italy. Maurizio Battaglia Parodi's major clinical and research interests include age-related macular degeneration, vascular diseases and dystrophies. He has taken part in many international clinical trials, and has authored over 210 peer-reviewed, scientific articles and three books. He has received many awards for his scientific activity and is a reviewer for some of the most prestigious international journals.

\section{Keywords}

Optical coherence tomography, imaging, EURETINA, diabetic macular oedema

Disclosure: Maurizio Battaglia Parodi has nothing to disclose in relation to this article. This is an expert interview and as such has not undergone the journal's standard peer review process.

Authorship: All named authors meet the International Committee of Medical Journal Editors (ICMJE) criteria for authorship of this manuscript, take responsibility for the integrity of the work as a whole, and have given final approval to the version to be published.

open Access: This article is published under the Creative Commons Attribution Noncommercial License, which permits any non-commercial use, distribution, adaptation and reproduction provided the original author(s) and source are given appropriate credit.

Received: 18 December 2017

Published Online: 21 December 2017

Citation: European Ophthalmic Review, 2017;11(2):90-1

Corresponding Author: Maurizio Battaglia Parodi, Department of Ophthalmology, Vita-Salute University, Ospedale San Raffaele, Via Olgettina 60, 20132, Milan Italy. E: maubp@yahoo.it

Support: No funding was received for the publication of this article.
$\mathrm{R}$ etinal disease is a major cause of poor vision especially in the ageing population, and age related macular degeneration is the main cause of vision loss in the developed world. ${ }^{1,2}$ Early recognition of the signs and symptoms of retinal disease can help prevent ophthalmologic complications and vision loss. It is unsurprising, therefore that retinal imaging and treatment of retinal disease is an active area of research. Founded in 2000, the European Society of Retina Specialists (EURETINA) is Europe's premier organisation for dissemination of research on retinal disease. In this expert interview, European Ophthalmic Review's Editor-in-Chief, Maurizio Battaglia Parodi, of the University Vita-Salute, Milan, Italy, discusses the highlights of the 17th EURETINA Congress in Barcelona, Spain on 7-10 September 2017, as well as the year's other major advances in retinal imaging technology and retinal disease.

\section{Q: In your opinion, what have been the most significant advances in retinal imaging technology in 2017?}

I think the most significant developments are regarding the latest advances in optical coherence tomography (OCT) resolution and its applications in the principal macular diseases. OCT angiography (OCT-A) is certainly the most promising non-invasive technique to study the microvascular structure of the retina and the choroid. Year after year, we have been observing technical implementations allowing for broader and clinically-oriented applications, along with quantitative analyses, including not only the classic vascular diseases, but also other retinal conditions, such as hereditary fundus dystrophies, Coats' disease and early alterations occurring in hypertension.

\section{Q: What were your highlights of this year's EURETINA congress?}

I have been particularly interested in the progresses coming from the gene therapy of chorioretinal dystrophies. For many years, we have been unable to propose any solution to patients affected by these debilitating conditions, but in light of the most recent results we can be more confident about the possibility to treat our patients in the next few years, at least in an attempt to stop the progression (e.g., choroideremia, Stargardt disease and retinitis pigmentosa). In this context, I believe that OCT-A might help unveil new therapeutic aspects and further improve the peri-operative planning.

\section{Q: What advances have been made this year in the use of long-acting and topical agents in the treatment of diabetic macular oedema?}

The use of long-acting and topical agents in the treatment of diabetic macular oedema (DMO) was certainly a key-point at EURETINA 2017, giving plenty of experts a platform to share their 
opinions. In particular, several groups from all over the world have shared their results and opinions regarding the use of lluvien ${ }^{\circledR}$ (fluocinolone acetonide intravitreal implant; Alimera Sciences, Alpharetta, GA, US). This approach seems fascinating, allowing the patients to achieve much longer treatment-free intervals, and optimal results can be achieved in patients characterised by chronic DMO, corresponding to DMO lasting at least 3 years. Nevertheless, I feel the need to express some concerns about its use, as the incidence of side effects, including cataract and increased intraocular pressure, is higher than other agents even in realworld setting. ${ }^{3}$

\section{Q: What advances have been made this year in the use of biomarkers to predict treatment response in DMO?}

The identification of biomarkers able to guide the therapeutic choice and to predict the final outcome is of the utmost importance. However, in spite of our efforts, we have not yet found reliable biomarkers, especially in choroidal neovascularization-related age-related macular degeneration and DMO. I hope that the new post-processing analyses which can derive from the OCT-A techniques, may help us identify different vascular patterns and thus guide our therapeutic approach.

\section{Q: What new developments in retinal disease and imaging can we look forward to in 2018?}

In my opinion, it is presumable that more in-depth investigations about the efficacy of new experimental therapies for retinal diseases (e.g., lluvien and gene therapy) will become available. Moreover, I am looking forward to seeing whether more reliable clinical applications can be gained from the use of OCT-A thanks to the development of new software, or the identification of more precise clinical biomarkers, in order to predict the response to the current treatments available, and thus to modify these therapeutic schemes. $\square$ 